\title{
Dialectical Interrelation Between: Problematic Situation, Real Problem, Scientific Problem, Object and Field, in the Investigation Design: A Necessary Reflection
}

\section{Interrelación Dialéctica Entre: Situación Prob- lémica, Problema Real, Problema Científico, Objeto y Campo, en el Diseño de una Inves- tigación: Una Reflexión Necesaria}

Science, Technology,

Entrepreneurship and

Innovation (SECTEI 2020)

Corresponding Author:

M. N. Navarro Ojeda

elyandy3@gmail.com

Published: 26 August 2021

Production and Hosting by

Knowledge $E$

(c) M. N. Navarro Ojeda

et al. This article is distributed under the terms of the Creative Commons

Attribution License, which permits unrestricted use and redistribution provided that the original author and source are credited.

\author{
M. N. Navarro Ojeda ${ }^{1}$, J. R. Pérez Pupo ${ }^{2}$, G. Barrazueta Rojas ${ }^{3}$, and M. A. \\ Batista Zaldívar ${ }^{4}$ \\ ${ }^{1}$ Escuela en Ingeniería de Industrias Pecuarias. Facultad de Ciencias Pecuarias, ESPOCH, \\ Riobamba, Ecuador \\ ${ }^{2}$ Escuela en Ingeniería Industrial. Facultad Ingeniería Mecánica, ESPOCH, Riobamba, Ecuador \\ ${ }^{3}$ Escuela de Control y Automatización, ESPOCH, Riobamba, Ecuador \\ ${ }^{4}$ Universidad Técnica de Manabí, Ecuador
}

\section{Abstract}

All research responds to the need of solutions to a problem that directly or indirectly affects society. The development of the same largely certifies and determines the constant search for solutions. The main objective of the present study was to arrive at a clear conceptualization, as complete as possible, of some the most important terms that come up during an investigation design: Problematic Situation, Real Problem, Scientific Problem, and Object and Field, given the visible shortcomings observed in pre- and post-graduate theses when the research work is being developed and defended. The methodology consisted of basically developing a reflection around these terms (the vision that different authors have in this regard was very helpful), as well as the presentation of varied examples contributed to see the meaning of these important concepts more clearly. The study shows in a scientifically grounded way the achievement of the above. The way in which the presentation of the different concepts was handled removes any possibility of mistakes.

Keywords: methodology scientific investigation, problema, object, field.

\section{Resumen}

Toda investigación parte del apremio en dar una respuesta a la solución de un problema que afecta de una manera directa o indirecta a la Sociedad. En gran medida, el propio desarrollo de la misma compulsa y determina esa constante búsqueda de soluciones. El objetivo principal del presente estudio fue llegar a una conceptualización clara, y lo más acabada posible, de algunos de los más importantes términos que se manejan durante el diseño de una investigación: Situación Problémica, Problema Real, Problema Científico, Objeto y Campo, dada las visibles falencias que se observan en tesistas de pre y postgrado a la hora de desarrollar y defender sus trabajos de investigación. La metodología consistió, básicamente, en desarrollar una reflexión en torno a estos términos; para lo cual resultó de gran ayuda la visión que tienen 
diferentes autores al respecto; así como, la exposición de variados ejemplos contribuyó a ver más definidamente el significado de estos importantes conceptos. El estudio muestra de forma científicamente fundamentada el logro de lo anterior. La forma en que se maneja la presentación de los diferentes conceptos aleja toda posibilidad de equívocos.

Palabras Clave: metodología investigación científica, problema, objeto, campo.

\section{Introducción}

Con demasiada frecuencia se observa, durante las predefensas y defensas de trabajos de titulación de los estudiantes de pregrado y otros de maestría, que existen evidentes dificultades para distinguir, deslindar, el problema real del cual se ha derivado su trabajo de investigación. La mayoría de las veces, se hace necesario recurrir a la hipótesis, y hacer 'ingeniería inversa', para dar con el mismo; y, en consecuencia, precisar el problema de investigación, el objeto y campo de acción. No resulta extraño, que al preguntárseles: ¿Cuál es el problema real? -del cual se ha derivado su problema de investigación-, se queden mirando desconcertados, tartamudeen y no sepan cómo precisarlo rápidamente, de forma inmediata, algunos ni siquiera logran verlo [1].

Existe un proverbio chino que asegura que: 'Cuando haya entendido el arte de la Estrategia, no hay una sola cosa que no pueda entender y pueda ver el camino de todas las cosas'. Y, en este orden, es donde la Metodología de la Investigación Científica y Experimental se torna en una herramienta de enorme trascendencia a la hora de dar solución a un problema; teniendo una concepción clara y muy bien definida acerca del término: problema y de otros importantes componentes de la misma. Cortés [2] define la Metodología de la Investigación Científica, como: 'Aquella ciencia que provee al investigador de una serie de conceptos, principios y leyes que le permiten encauzar de un modo eficiente y tendiente a la excelencia el proceso de la investigación científica'; un concepto similar es el que expresa Rodríguez [3].

Existe una coincidencia total, por muchos autores, en el planteamiento siguiente, que constituye un verdadero axioma de la lógica más elemental: 'El problema existe independientemente posea o no solución; no se fabrica, solo existe' [4, 5]; a pesar de ello, no todos logran verlo. Y este problema puede tener varias vías de solución: p.ej.: puramente ingenieril: como cuando se proyecta una máquina, equipo o partes de estos; o, por el contrario, haya que recurrir a la búsqueda de nuevos caminos no explorados total o parcialmente; es decir en la concepción de nuevas teorías que puedan avalar la solución de éste. 
Con todo y lo que está desarrollada la ciencia hoy día, aun el hombre tendrá que esperar a estadios superiores, para encontrar solución a muchos de los problemas que lo agobian actualmente en disimiles ámbitos de la vida social y científica; y en no pocas veces, se logra llegar a obtener un curso exitoso en las investigaciones. Martínez [6], citando al eminente físico y filósofo austríaco Erwin Rudolf Josef Alexander Schrödinger -adjudicado con el premio Nobel, por su importante descubrimiento de la ecuación fundamental de la mecánica cuántica (base de la física moderna), y sus notables contribuciones también en la termodinámica, plantea que: 'La ciencia actual nos ha conducido por un callejón sin salida y que la actitud científica ha de ser reconstruida, que la ciencia ha de rehacerse de nuevo' (p. 2).

Retomando la reflexión inicial, sobre esta problemática en la formación de los egresados, surgen algunas preguntas: ¿Qué está fallando? ¿Es que las causas de estas deficiencias están en el proceso de enseñanza-aprendizaje en pregrado?; inclusive, ¿es que también no se encuentran presentes -en un número nada despreciableen trabajos de investigación de postgrado, contextualizadas en tesis de maestrías, e inclusive de doctorado? Pero además, es una problemática que se observa en no pocos países. Éstas, son preguntas que no son fáciles de contestar; pero, la realidad es una: ¡existe! Al respecto, Morrillo [7], da una definición muy acertada y pragmática cuando enfatiza que la ejecución de una tesis de grado conlleva el siguiente compromiso:

Es una labor de gran importancia para la formación académica y profesional de la persona que la elabora, al demostrar éste el manejo de técnicas y metodologías de investigación, así como conocimientos profundos sobre estudios, según el grado o título académico a optar (licenciatura, maestría o doctorado) (p. 920).

Lógicamente, toda investigación parte de un problema real, latente éste dentro de una situación problémica, del cual se derivan -en muchas ocasiones- muy diversos planteamientos de problemas científicos -con la aplicación de métodos científicos-, que permiten dar solución parcial o total al mismo; a la necesidad que engendra su solución; al mismo tiempo, la declaración de los objetivos es parte fundamental, pues precisa las metas, propósitos o propuestas hacia la consecución del resultado final. Normalmente, en la mayoría de los trabajos de investigación, los objetivos aparecen inmediatamente después de declarado el problema científico. Aunque, sería interesante ver, como, en determinadas situaciones complejas, los objetivos específicos, pueden engendrar nuevos problemas científicos a resolver.

No obstante, todo debe comportarse como una integral por partes: en múltiples situaciones un problema real demanda del concurso de un equipo multidisciplinario, tal y como afirma Gómez [8, p. 29]; el cual desempeñará muy variadas tareas, específicas; mismas que pueden estar dando respuesta a objetivos y planteamientos científicos diferentes, pero concurrentes todos en la solución del problema real; asociados a hipótesis de trabajo diferentes, también específicas (correspondiente, cada una o varias, 
a un problema científico determinado); póngase el solo ejemplo del diseño o rediseño de un avión, o de una maquinaria agrícola compleja, etc., donde son múltiples los retos a superar en la armonía constructiva y de funcionamiento de los muy disimiles subsistemas, donde la dialéctica va imponiendo el ritmo y dirección de la investigación. La investigación vista como un proceso, donde la aplicación del método científico, cuyos modelos pueden ser muy variados, -ver p. ej. Ávila [9], cuando hace una descripción de la versión moderna del método de Popper que la representa en forma de dos modelos circulares: La Rueda de Wallace y la Espiral de Leedy (p. 11), que marcan el paso y rigurosidad de la misma.

Arias [10] lo define simplemente como: ‘El conjunto de pasos, técnicas y procedimientos que se emplean para formular y resolver problemas de investigación mediante la prueba o verificación de hipótesis' (p. 19). Esta definición está muy cercana, a la que expresa Cerda (2000), citado por Niño [11], cuando escribe que: 'O sea requerimos la ayuda de un método, de un camino, de un ordenamiento y de procedimientos sistemáticos que nos faciliten el proceso y el acto de conocer' (p. 26).

Igual razonamiento se aplica en la determinación del Objeto y el Campo: al macro y micro universo respectivamente. $Y$, siempre tener muy presente, durante el diseño, planteamiento, estructuración o concepción de toda investigación, observar, con el mayor cuidado, la interdependencia 'eslabonada' de los diferentes componentes que integran el diseño de una investigación.

El presente trabajo, en apretada síntesis, pretende dar una visión, lo más simplificada posible, acerca de conceptos bien diferenciados, pero no tan bien comprendidos: La situación problémica, el problema real y el problema científico imbricados totalmente a los objetivos, al objeto y al campo de acción, en el desarrollo de una investigación.

\section{Metodología}

En la búsqueda de establecer una conceptualización clara en la interrelación dialéctica de los términos: Situación Problémica, Problema Real, Problema Científico, Objeto y Campo, en el diseño de una investigación, se hizo necesario la realización de una profusa revisión bibliográfica y valiosas consultas de expertos, acerca de esta interesante temática; lo cual propició obtener una visión más nítida de la interdependencia de cada uno de estos importantes conceptos.

De gran ayuda resultó la exposición de diferentes ejemplos que ilustraron de manera gráfica y bien definida la conceptualización de los referidos términos.

Después de la necesaria revisión bibliográfica, y como complemento indispensable, se expone una información complementaria acerca del muy controvertido e importante término: problema, visto a través del criterio de prestigiosos autores. 


\section{Resultados y Discusión}

Se comentaba inicialmente, que un buen número de estudiantes e investigadores no sabían precisar el problema real de su investigación, que no sabían concretarlo. Entonces: ¿Cómo es posible iniciar una investigación si no se conoce claramente a qué necesidades de solución responde?; ¿cuál es el problema real que está demandando ser resuelto?

\subsection{Estructura metodológica en el desarrollo del diseño de una investigación. Conceptualización de la situación problémica}

Todo parte de una Situación Problémica. En la Figura 1, se puede observar la estructura metodológica en el desarrollo del diseño de una investigación. El diseño de la investigación visto, según Arias [10], como: 'La estrategia general que adopta el investigador para responder al problema planteado' (p. 27).

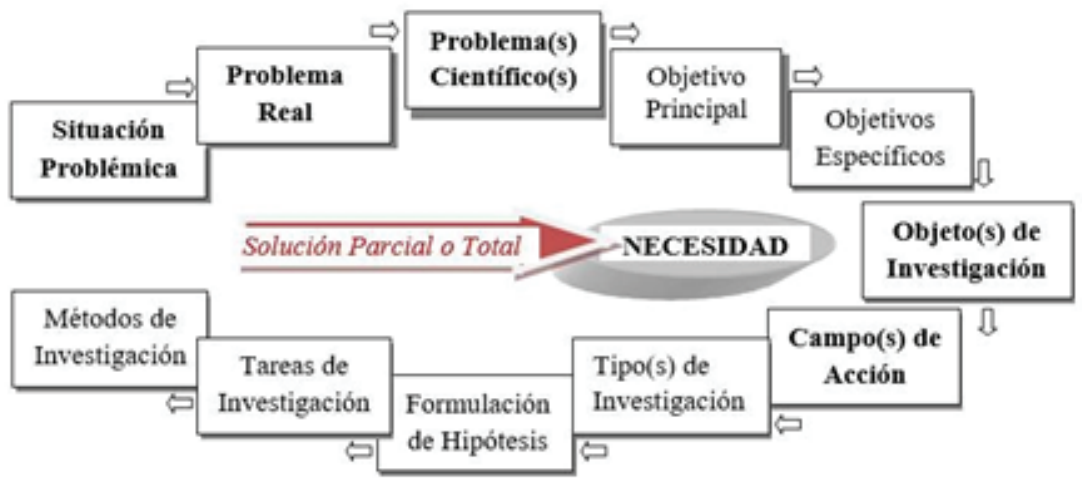

\section{Figure 1}

Diseño, planteamiento, estructuración o concepción de una investigación.

Sin lugar a dudas, es un hecho imprescindible tener una clara noción acerca del concepto: 'Situación Problémica'. La Figura 2 explica por si sola este importante concepto, en el caso de un síndrome de Listeriosis, producida esta mortal enfermedad por la bacteria Listeria monocytogenes [12,13]. Tal y como se puede observar, la Situación Problémica informa de un conjunto de signos, características o aristas de un fenómeno determinado; todo lo cual se resume en otro importante concepto: 'el Problema Real'.

Otro ejemplo gráfico que informa de una Situación Problémica se puede ver en la Figura 3, donde se presentan las falencias en la fabricación de la cerveza IPA artesanal. Si se pudiera hablar de una 'hipérbole literaria gráfica', o quizás de una metáfora, la mencionada figura traduce un conjunto de características entremezcladas, girando en un sentido y delimitando claramente el vórtice de un fenómeno: el Problema Real. 


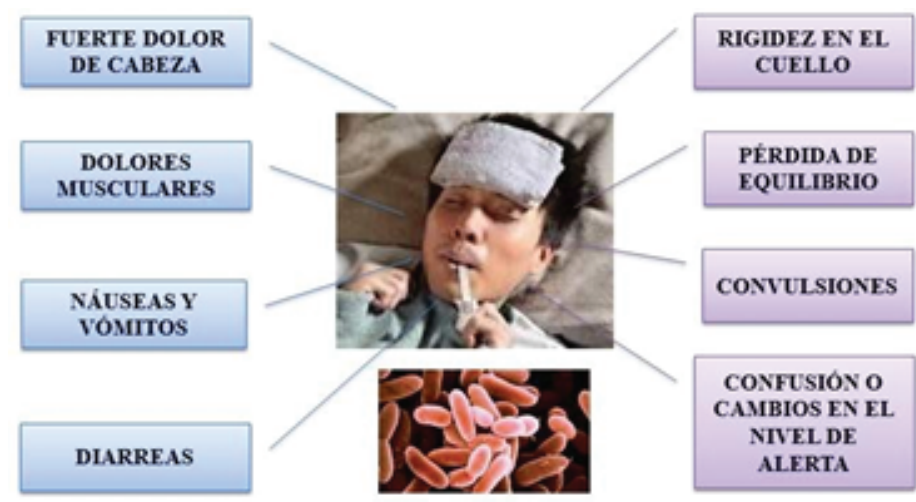

\section{Figure 2}

Signos que pueden estar presentes en un síndrome de Listeriosis.

Indefectiblemente, hay que sumergirse dentro de la Situación Problémica y de esta forma ver el Problema Real que subyace en el centro de la misma. Como en el caso de los ciclones tropicales, no solamente es suficiente observar el fenómeno desde afuera, sino introducirse en el vórtice para verlo desde adentro, y llegar a su esencia misma.

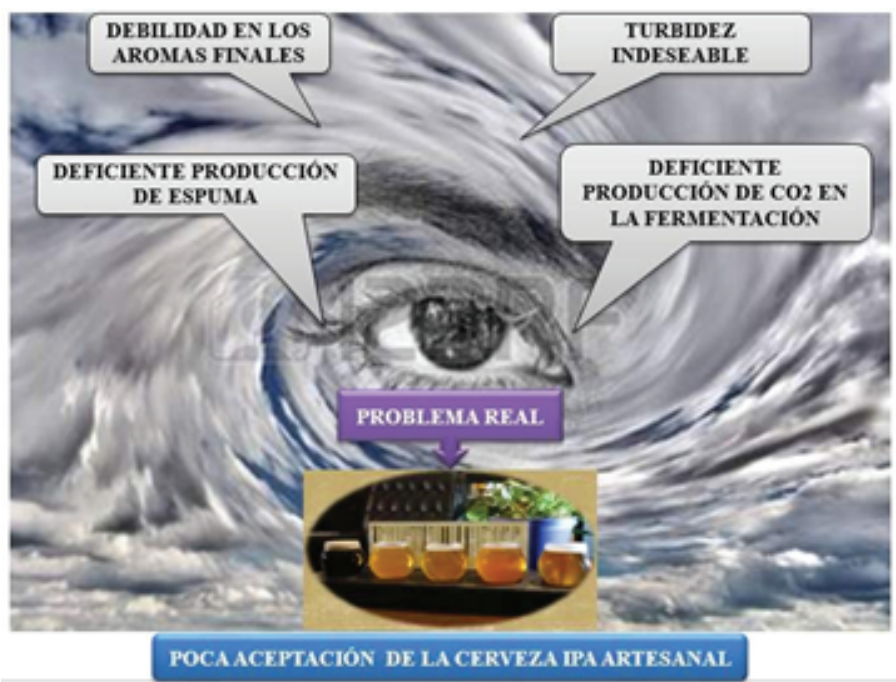

Figure 3

Situación problémica presente en la fabricación de la cerveza IPA artesanal.

\subsection{Correspondencia entre el problema y sus causas - relación: efecto-causa: efecto causa-raíz. Problema real y problema de investigación}

Al tratar de reflexionar sobre este aspecto, acuden nuevas interrogantes:

¿Cuál es la raíz de todos estos males?; ¿se enseña a investigar, utilizando correctamente las herramientas metodológicas establecidas, a cada pupilo? 
Sin ir a la enseñanza de pregrado tratando de buscar las causas, pueden tratarse diferentes situaciones desde el presente. Realmente, los problemas metodológicos pueden ser muy diversos; póngase el caso que a un estudiante se le dé solo el tema o Problema Científico - objeto de su trabajo de investigación- sin explicarle su origen, de cual Problema Real se deriva (El término Problema Científico es el más usado - ver p.ej., lo planteado por Pérez Alcolea [14, p. 4]; algunos prefieren Ilamarlo Problema de Investigación o simplemente Problema). Indicarle, por ejemplo, que se ocupe del siguiente Tema: 'Desarrollo de una metodología para la selección de las piezas de repuesto de una máquina cosechadora, durante las reparaciones operativas y profilácticas'.

El estudiante puede que desarrolle una buena metodología, pero quizás no haya interiorizado que ese problema de investigación, del cual él se ocupó, o se está ocupando, está derivado de la necesidad de resolver un problema mayor, el Problema Real; que, perfectamente pudiera ser: El bajo rendimiento de las máquinas (dependiente éste de varias causas: desde la calificación del operario, la fiabilidad de la máquina, hasta el oportuno abastecimiento de las piezas de repuesto cuando se necesiten).

Existe una correspondencia innegable entre el problema y sus causas - relación: Efecto-causa-; efecto: 'causa raíz', que, a su vez, éstas pueden convertirse en nuevos problemas, todos reales, en una derivación de los niveles de actuación o dependencia. En la Figura 4 se muestra un ejemplo de lo antes dicho: La dependencia del problema real de varias causas; convirtiéndose éstas, a su vez, en nuevos problemas -más específicos-, a resolver. El problema es lineal, consecutivo, en una interrelación dialéctica; causa -raíz.

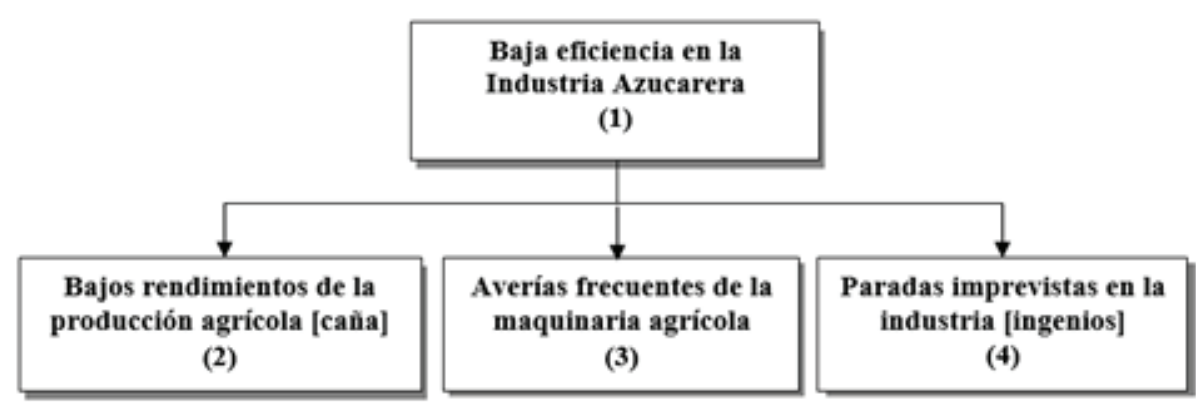

\section{Figure 4}

Relación: Problema - Causa - Problema.

En este ejemplo, se puede observar que el Problema Real (1) se conecta a tres posibles causas; directamente relacionadas a: la parte agrícola y a la maquinaria agrícola e industrial; que, al mismo tiempo, constituyen también problemas reales, vistos desde una perspectiva más específica. 
Pero, más interesante aún es constatar que esas causas-problemas: (2), (3) y (4), están directamente relacionadas a nuevas causas, que se convierten a su vez en nuevos problemas (6), (7) y (8). Ver ejemplo en la Figura 5.

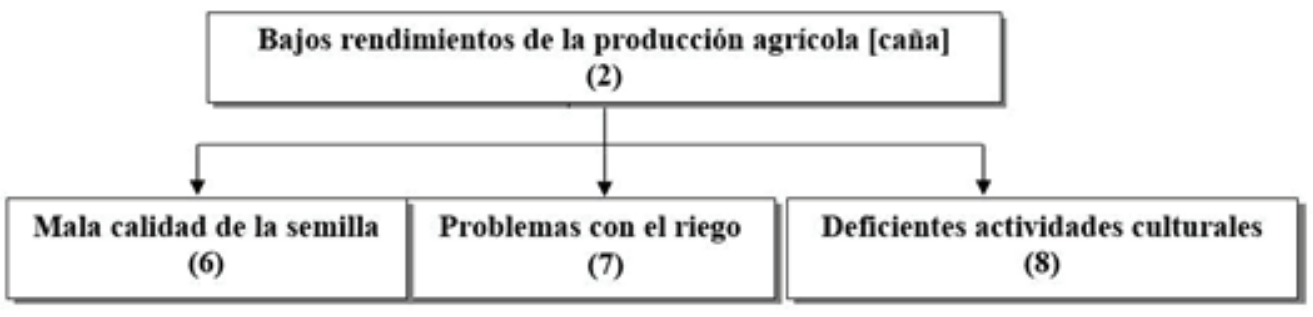

\section{Figure 5}

Segundo nivel en la relación: Problema - Causa - Problema.

Este razonamiento puede continuar hasta determinar todas las causas raíces de cada problema intermedio derivado del problema real inicial: Es, sencillamente, una simple concatenación de relaciones causa-efecto.

Estos problemas reales (derivados), todos, pueden generar diferentes investigaciones -Problemas Científicos, como se apuntara anteriormente-; dependiendo estos últimos de tres importantes aspectos, los recursos: humanos, materiales y financieros; y no menos importante, un cuarto factor: El estado de desarrollo alcanzado por la ciencia y la tecnología hasta esos momentos.

Semejante relación dialéctica: Problema-causa-problema-problema de investigación, es la misma que se observa en la relación objeto y campo de investigación.

\subsection{Correspondencia biunívoca entre el objeto y el campo. Mutación en su comportamiento dialéctico}

Ante todo, sería conveniente delimitar el término: Objeto de investigación; Behar [15] lo conceptualiza como; 'Es consecuencia del planteamiento del problema, delimita aquella parte de la realidad que interesa estudiar' (p. 24). Existe una correspondencia biunívoca entre el Objeto y el Campo, entre el macro y el micro universo que representan: susceptibles al planteamiento de similares derivaciones, como las ya observadas.

Un Objeto no tiene que ser necesariamente un ente físico, como muchos creen, sino que el mismo puede ser una fórmula, cualquier modelo matemático o de procesos, un software, etc.). Inclusive, de acuerdo a los objetivos programados, -y recursos disponibles-, un determinado Campo de Acción puede convertirse en Objeto de Investigación, que, a su vez, podría contener uno o más campos de acción.

Tómese p.ej., la elevación de la eficiencia o fiabilidad de una máquina cosechadora como objeto de investigación -ver el trabajo desarrollado por Navarro et al. [16]- y dirija su atención a su sección receptora (visto, según una visión estructural, ésta podría ser el 
campo de acción); pero, si se toma esta última como objeto de investigación, el campo puede ser el estudio de los discos de corte; al mismo tiempo, los discos de corte ver el trabajo desarrollado por Daquinta et al. [17]- pueden convertirse en objeto y las cuchillas en campo de acción, a través de: el diseño -cambio de su geometría o empleo de nuevos materiales-, o la evaluación de sus características técnico explotativas; o la determinación del módulo de piezas de repuesto de las cuchillas necesarias para una zafra, etc., respectivamente: es decir, según sea el objeto asociado: máquina, sección receptora, disco de corte, cuchillas: el (los) campo(s) de acción siempre quedarán delimitados, supeditados, al Objeto de Investigación.

Puntualizando: La relación: objeto-campo, campo-objeto, objeto-campos, es la misma observada anteriormente en la relación: problema real-problema de investigación; pues dichas relaciones están interconectadas, imbricadas, en una misma relación de interdependencia: existe un problema de investigación porque existe un problema real; y éste, a su vez, lógicamente, pertenece, o se encuentra, en un determinado objeto; que, finalmente, -el Problema Real-, será susceptible a ser resuelto parcial o totalmente, a través de diferentes vías, o campos de acción, según los objetivos propuestos.

\subsection{Concatenación de las relaciones existentes entre el prob- lema real y los objetivos propuestos. Mutación en su com- portamiento dialéctico}

Otra arista del problema sería analizar la concatenación de las relaciones existentes entre el Problema Real y los Objetivos propuestos -que se transforman en problemas a resolver: Posibles problema(s) científico(s); y, que a su vez, son contentivos de nuevos objetivos derivados.

El ejemplo, que aparece a continuación, muestra estas relaciones. Obsérvese como, secuencialmente, un determinado Objetivo Específico, puede transformarse en un Problema Científico; es por ello que se justifica la aparición de los grupos multidisciplinarios para poder dar respuesta a cada uno de los objetivos específicos propuestos, que conduzcan al cumplimiento del principal.

Para mayor claridad, véanse en las Figuras 6-8, en ese orden, el Problema Científico: ¿¿Las condiciones edafoclimáticas y del relieve son factibles para la construcción del trasvase E-O?', parte de un objetivo específico mayor a cumplimentar: 'Estudio de las condiciones edafoclimáticas y del relieve de la región', derivado del problema científico: ‘Cómo concebir un sistema eficiente de trasvase de agua E-O?’; que a su vez, se deriva de la necesidad de dar solución al problema principal: 'La sequía y su repercusión en la vida social y económica de la provincia Holguín'.

Posteriormente, las Figuras 9 y 10 completan el planteamiento del diseño de la investigación, con la concepción bien definida de la(s) hipótesis, teniendo especial 


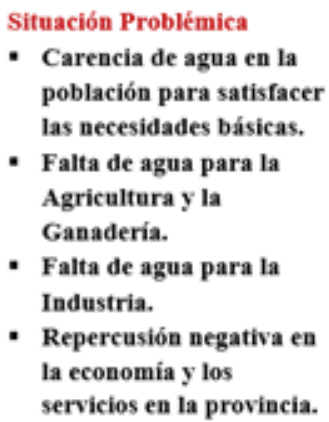

\begin{tabular}{|l|l|}
\hline \begin{tabular}{l|l|} 
Problema Real \\
La sequía y su \\
repercusión en \\
la vida social y \\
económica de \\
la provincia
\end{tabular} & $\begin{array}{l}\text { Objetivo } \\
\text { Principal } \\
\text { Eolguínación. }\end{array}$ \\
\cline { 2 - 2 } & \\
\hline
\end{tabular}

Objetivos Específicos

1. Estudio de las principales causas que posibilitan la pérdida de agua.

2. Concepción de un sistema effciente de distribución de agua para la población, industria $y$ agricultura.

3. Concepción de un trasvase Este-Oeste (E-O).

Figure 6

Descripción de la situación problémica y planteamiento del problema real y objetivos de la investigación.

\begin{tabular}{|l||l|}
\hline Problema Científico & $\begin{array}{l}\text { Objetivo Principal } \\
\mathrm{PC}\end{array}$ \\
oEs $\mathrm{PR}$ & oE $\mathrm{PR}$ \\
¿Cómo concebir un & Estudio de la \\
sistema eficiente de & factibilidad técnico \\
trasvase de agua E-O? & $\begin{array}{l}\text { económica en la } \\
\text { concepción del trabase } \\
\text { E-O. }\end{array}$ \\
\hline
\end{tabular}

Objetivos Específicos PC OE 3 PR

1. Determinación de la posible ruta del trasvase E-O.

2. Estudio de las condiciones edafoclimáticas y del relieve de la región.

3. Determinación de los recursos humanos, materiales y financieros necesarios.

\section{Figure 7}

Planteamiento del problema científico derivado del objetivo específico 3 , del problema real.

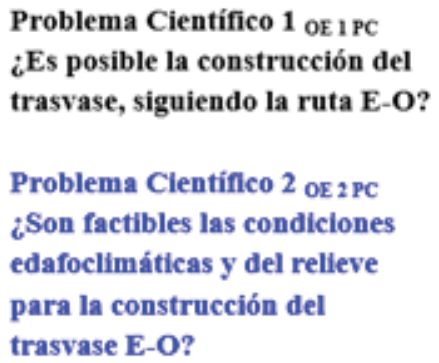

Objetivo PrincipaloE2 PC OE 3 PR

Determinación de las condiciones

edafoclimáticas y del relieve de la región.
Objetivos Específicos OE2 PC OE 3 PR

1. Determinación de las principales cuencas hidrográficas: ríos, lagos, embalses.

2. Estudio del $\%$ de precipitaciones anuales históricas $\mathbf{y}$ actuales de la región.

3. Estudio de los suelos y relieve de la región: principales elevaciones y llanos, etc., en la posible ruta.

\section{Figure 8}

Planteamiento del problema científico derivado del objetivo específico 2, correspondiente al problema científico derivado del objetivo específico 3, del problema real.

cuidado en el manejo de las variables dependientes e independientes, en su relación causa-efecto. Otro importante eslabón es la planificación de las tareas y posibles métodos de ejecución. En este contexto, las tareas deben ser planificadas de forma muy objetiva, teniendo en cuenta los recursos disponibles y los plazos de ejecución. La realización de la investigación debe estar sustentada, en todo momento, por métodos 
científicamente fundamentados, ya sean estos: teóricos, empíricos o estadísticos, o simplemente una combinación de todos ellos.

\begin{tabular}{|c|c|}
\hline $\begin{array}{l}\text { Objeto de la } \\
\text { Investigación } \\
\text { Estudio de las } \\
\text { cuencas } \\
\text { hidrográficas y } \\
\text { relieve de la } \\
\text { región. }\end{array}$ & $\begin{array}{l}\text { Campo de Acción } \\
\text { Estudio de las } \\
\text { cuencas } \\
\text { hidrográficas y } \\
\text { relieve de la región } \\
\text { para la construcción } \\
\text { del trasvase E-O. }\end{array}$ \\
\hline
\end{tabular}

\begin{tabular}{|l|}
\hline Tipo de \\
Estudio \\
Explicativo \\
\hline
\end{tabular}

Hipótesis: HI1: existen suficientes fuentes de abasto de agua en la región HI2: el relieve es favorable para la construcción del trasvase E-O.

\section{Figure 9}

Planteamiento del objeto y campo de investigación, tipo de estudio y posibles hipótesis del Problema Científico 2OE 2 PC.

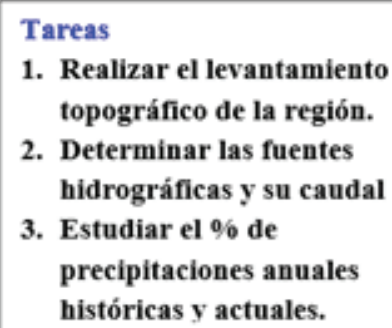

\section{Métodos}

Teóricos:

- El Análisis y la Síntesis

- La Inducción y la Deducción

- Método Dialéctico, Histórico, etc. Empíricos:

- Método de Observación Cientifica

- El Método de la Medición, etc. Estadísticos:

- Determinación de la muestra

- Tabulación de datos

- Aplicación de métodos de la estadística matemática.

Figure 10

Planteamiento de posibles tareas y métodos relacionados al Problema Científico 20 E 2 PC.

\section{5. ¿Cómo definen los términos: problema y problema de investi- gación algunos autores?}

Finalmente, resulta de mucho interés ver lo que dicen algunos autores acerca de los términos: problema y problema de investigación.

Álvarez [18] plantea que:

El proceso se inicia en torno a un área problemática de investigación, éste surge de un contexto teórico o práctico. Esta etapa puede sintetizarse en la pregunta ¿De qué se trata? La elección del problema es de índole personal; depende de los intereses del investigador: comprobar teorías, descubrir o generar conocimiento o mejorar y optimizar la práctica. El planteamiento del problema supone el inicio de la investigación, aunque no tiene que ser necesariamente la única manera. Esta surge cuando tenemos que dar respuesta a una pregunta o dificultad que consideramos problemática. 
Mientras que Córdova [4], refiriéndose a las Ciencias sociales, puntualiza: Se entiende por problema una situación que favorece la aparición y la agudización de dificultades sociales entre ellas las de carácter pedagógico, o que limita o entorpece el desarrollo normal de los procesos. El problema se presenta como una contradicción formada por dos elementos internamente relacionados. El problema no se inventa, se formula a partir de las contradicciones de la propia realidad, se parte de situaciones problémicas: hechos, acciones reiterados que conspiran contra el normal desarrollo de la comunidad, del proceso docente educativo o de los procesos en general.

Cruz [19] se proyecta en su definición recurriendo a las características de la situación problémica:

El problema de investigación: se expresa en términos concretos y explícitos a través del planteamiento y la formulación ... Planteamiento del problema: descripción de la situación actual que caracteriza el objeto de conocimiento (síntomas y causas, diagnóstico). Identificación de situaciones futuras al sostenerse la situación actual (pronóstico). Presentación de alternativas para superar la situación actual (control al pronóstico) ... Formulación del problema: parte de la identificación y descripción de los síntomas que se observan y son relevantes en la situación, relacionados con las causas que lo producen, el diagnóstico.

Muy pertinente es el llamado que hace Díaz [20] en la diferenciación que se debe hacer entre el problema de investigación y su situación problémica. Evidentemente, y tal y como se planteó inicialmente, existe una derivación de conceptos y acciones: Primero existe el Problema Real (contextualizado en la Situación Problémica), para que, después, se deriven de él posibles Problemas Científicos.

La inmensa mayoría de las personas identifica el problema de investigación con la situación problema o la formulación problemática pagando por ello un elevado precio que se traduce en pérdida de tiempo, de recursos mentales, materiales, financieros $y$, lo que es más lamentable, el fracaso en el proceso investigativo.

Rodríguez et al. [3], por su parte, plantean el problema de investigación como una interrelación que se establece entre el Objeto y el Sujeto, sobre el puente de la necesidad de dar solución al Problema Real, a través del Problema Científico, visto éste como la expresión de los límites del conocimiento científico, en relación al estado de desarrollo de la ciencia y la tecnología hasta esos momentos.

El problema, (el por qué), de la investigación, lo podemos definir como la situación propia de un objeto, que provoca una necesidad en un sujeto, el cual desarrollará una actividad para transformar la situación mencionada y resolver el problema ... es objetivo en tanto es una situación presente en el objeto; pero es subjetivo, pues para que exista el problema, la situación tiene que generar una necesidad en el 
sujeto... se manifiesta externamente en el objeto y es consecuencia precisamente del desconocimiento de elementos y relaciones que existen en el mismo. El planteamiento del problema científico es la expresión de los límites del conocimiento científico actual que genera la insatisfacción en el sujeto: la necesidad.

La solución del Problema Real se traduce mediante el Problema Científico, enmarcado éste dentro de una determinada idea. La idea, tal y como Hernández Sampier [21] la concibe, es el eslabón de partida para dar solución al problema. Pero, llegar hasta su estructuración, afinación, y una clara delimitación de sus posibles alcances, conlleva, la mayoría de las veces, a un prolongado tiempo de revisión bibliográfica y consulta de expertos. Ya, sobre esas bases potencialmente firmes, se puede dar continuidad a los subsiguientes pasos en el desarrollo de la investigación.

Afinar y estructurar más formalmente la idea de investigación ... Como señala Ackoff (1953), un problema correctamente planteado está parcialmente resuelto, a mayor exactitud corresponden más posibilidades de obtener una solución satisfactoria ... El tiempo que pudiera mediar entre la Idea y el Planteamiento del Problema es totalmente relativo; puede ser inmediato, o por el contrario llevarse a cabo en un tiempo prolongado Sampier.

Sierra [22] se refiere al concepto 'Problema' imbricado en el proceso de Investigación Científica. De hecho ya él parte de la existencia de un Problema Real, como una necesidad latente de dar solución al problema.

El estudio analítico de la práctica de los procesos investigativos evidencia que todo proceso de investigación científica está orientada a la solución de problemas científicos. Todo problema científico se formula conscientemente y su solución debe ser alcanzada en el curso de la investigación. En consecuencia, la primera característica del proceso de Investigación Científica será el concepto de problema.

Mientras que Tapia [23] coincide con Hernández Sampier [21] en la formulación del Problema de Investigación, partiendo de una determinada Idea, cuando plantea que: 'La formulación del problema de investigación es la etapa donde se estructura formalmente la idea de investigación, es este el primer paso, donde se define qué hacer'.

En su trabajo 'Notas de Clase para la auto preparación sobre conceptos básicos de los elementos componentes del diseño de la investigación y generalidades del diseño del experimento', Carrasco [24], en apretada síntesis, expone el criterio que expresan otros autores, acerca del término: 'Problema'.

Y más adelante, el propio Carrasco [24], citando a otros autores puntualiza: El problema es el punto de partida de toda investigación, puede llegar a ser la etapa más larga del proceso de investigación, y surge por una laguna en el conocimiento, por una aparente contradicción respecto a investigaciones anteriores, o simplemente por observar un fenómeno nuevo (Bizquera). 
El problema científico conduce a la formulación del anteproyecto de la investigación, por lo tanto, da origen a la investigación, motiva la predicción de nuevas situaciones y la transformación de la realidad para mejorar la calidad de vida de los involucrados en el mismo (Ernesto Hernández).

Si no tienes un problema, entonces no necesitas un proyecto. Los proyectos son acontecimientos para resolver problemas... (Anthony Davies).

Se puede observar que existe una misma línea de pensamiento hacia estos conceptos; algunos lo esbozan o definen de una forma más directa y otros más velada, pero todos coincidentes

\section{Conclusiones}

Tal es la importancia de la Metodología de la Investigación Científica y Experimental, que es asignatura obligada en mayas curriculares o planes de estudio de todas las carreras universitarias, maestrías y doctorados. Es una herramienta, que como la computación, posee un lugar demasiado necesario, como para no ser consultada. Toda investigación surge con la aparición o detección de un problema: de un problema real, que no siempre, como en el problema científico, debe estar supeditado al aspecto subjetivo de la conciencia del hombre en la satisfacción de una necesidad transitoria, en un determinado momento histórico del desarrollo social o tecnológico.

Sobre este último aspecto es válida la siguiente reflexión: existen problemas que, por su naturaleza, desbordan este estrecho marco de lo subjetivo. Problemas de salud, tales como el VIH, el cáncer, etc., aún no han encontrado una solución definitiva; y constituyen retos insoslayables para la comunidad científica. Pueden citarse también otros problemas similares en el campo de las ingenierías, donde aún la eficiencia, la durabilidad y otros índices de fiabilidad, funcionamiento y explotación de máquinas y equipos están muy lejos de satisfacer las necesidades actuales; o citar el muy real fenómeno de las desbastadoras sequias que exterminan plantas y animales, incluyendo al hombre. ¿Es que acaso ya la humanidad ha encontrado y aplicado globalmente una fórmula para exterminar el hambre y las diferencias entre países, y ricos y pobres? ¿Y dónde situar, en esta larga lista de asuntos pendientes, el calentamiento global, responsable del efecto invernadero; la devastación de extensísimas áreas boscosas, pulmón del planeta y, en muchos casos, tornadas en extensas áreas desérticas; la contaminación ambiental de la atmosfera, lagos, ríos y del océano mundial?

Y, a pesar que el hombre explora nuevos caminos, y en este contexto, las nanociencias y nanotecnologías ocupan un lugar preponderante; ya que con el estudio del control y manipulación de sistemas cuyo tamaño es de unos pocos nanómetros respectivamente, se puede llegar a soluciones increíbles, especialmente en la medicina y en su aplicación técnica y tecnológica de múltiples ramas. A pesar de todo ello, no 
solamente la comunidad científica debe cargar con la responsabilidad en la búsqueda de soluciones de todos estos males, sino que se requiere, por una parte, de voluntades políticas y, por la otra, de la concientización de la población mundial, que mancomunadas todas las acciones, coadyuven y conduzcan a la aplicación de medidas radicales e irreversibles conducentes a convertir el planeta en un lugar equitativo, ponderado y sustentable.

En resumen: El problema es el punto de partida de toda investigación: precisar el problema real y el científico correctamente -pueden ser varios, en dependencia de la complejidad de lo tratado y de los objetivos y recursos disponibles-, puede llegar a ser la etapa más larga de todo el proceso de investigación; pues únicamente se logra con la afinación, la adecuada estructuración de la investigación que ha de realizarse; y, solo después, de haber consumado una exhaustiva revisión bibliográfica y consulta de expertos.

\section{References}

[1] Ojeda MN. ¿Cuáles son los cuatro momentos esenciales a tener en cuenta en toda investigación científica? Revista CTA. 2009;18(4):79-81.

[2] Cortés M, León MI. Generalidades sobre Metodología de la Investigación. Colección Material Didáctico. $1^{\text {st }}$ edition. México: Universidad Autónoma del Carmen; 2004.

[3] Expósito FR, García RC, Pupo MG. Metodología de la Investigación. Facultad de Informática Matemática; 2008.

[4] Martínez CC. Consideraciones sobre Metodología de la Investigación. Universidad de Holguín; 2007.

[5] Ojeda, NM. Ciclo de conferencias sobre Metodología de la Investigación Experimental, del Programa de Doctorado Curricular, impartido en el Instituto Universitario Experimental de Tecnología de la Victoria, Edo. República Bolivariana de Venezuela: Aragua; 2008. 162 p.

[6] Miguel M. La Investigación Cualitativa (Síntesis Conceptual). Revista IIPSI. 2006;9(1): $123-146$.

[7] Moreno M. Labor del tutor y asesor de trabajo de investigación. Experiencias e incentivos. Educere. 2009;13(47):919-930.

[8] Marcelo G. Introducción a la Metodología de la Investigación Científica. $1^{\text {st }}$ edition. Córdova:, Argentina. Disponible en: https://books.google.es/ books?hl=es\&lr=\&id=9UDXPe4U7aMC\&oi=fnd\&pg=PA9\&dq=metodologia+de+ la+investigacion\&ots=b8qNK-mLDT\&sig=loNHXnicRcuFdOcPz5ikqixJJI8\#v= onepage\&q=metodologia\{\%\}20de\{\%\}20la\{\%\}20investigacion\&f=false

[9] Baray AH. Introducción a la Metodología de la investigación. 2006. Available from: www. Eumed.net/libros/2006c/203/. 
[10] Arias F. El proyecto de Investigación: Introducción a la Metodología Científica. $7^{\text {th }}$ Edición. Caracas, Venezuela. Disponible en: https://books.google.es/ books?hl=es\&lr=\&id=W5nOBgAAQBAJ\&oi=fnd\&pg=PA11\&dq=metodologia+de+ la+investigacion\&ots=kXpQfrrpo9\&sig=tzbDUztAXGFeoz9IVMRzGNDBWL4\#v= onepage\& $q \& f=$ false

[11] Rojas MN. Metodología de la Investigación. Diseño y ejecución). Bogotá: 2011., Colombia. Disponible en: https://books.google.es/books?hl=es\&lr=\&id= CyejDwAAQBAJ\&oi=fnd\&pg=PA15\&dq=metodologia+de+la+investigacion\&ots= V5X4kHas2C\&sig=dM5x9PXAsxUblYUpQX8GTWJwc6E\#v=onepage\&q\&f=false

[12] Allerberger F, Wagner M. Clinical microbiology and infection. 2010;16(1):16-23.

[13] Swaminathan B, Gerner-Smidt P. The epidemiology of human liisteriosis. Elsevier. 2007;9(10)1236-1243.

[14] Alcolea PS. Dinámica integradora de la formación del conocimiento científico pedagógico en el contexto de las tecnologías de la información y la comunicación [Doctoral Thesis]. Santiago de Cuba: Universidad De Ciencias Pedagógicas "Frank País García"; 2015.

[15] Rivero BD. Metodología de la Investigación. 2008. Available from: $\quad$ http://www.rdigital.unicv.edu.cv/bitstream/123456789/106/3/ Libro\{\%\}20metodologia\{\%\}20investigacion\{\%\}20este.pdf

[16] Navarro J, Martínez J, Tamayo R, Sánchez R. Determination of exploitative technological indicators of the sugarcane harvester prototype CCA-5000. Revista trimestral. 2019;25(1).

[17] Daquinta L, Bonilla J, Recarey C. Desempeño de los segmentos de corte base en las cosechadoras de caña de azúcar Case-IH 7000 Revista Ciencias Técnicas Agropecuarias. 2018;27(3)., E-ISSN: 2071-0054. Disponible en: file:///C:/Users/User/ AppData/Local/Temp/982-3109-1-PB.pdf

[18] Valdivia IA. Facultad de ciencias Sociales y Humanísticas. Dpto. Psicología. Universidad Central de Las Villas, 1997.

[19] Silvia C. Metodología de la Investigación. Universidad de Oriente; 1998.

[20] Caballero, JRD. De la situación problemática al problema de investigación. 2010.

[21] Sampier RH, Fernández CC, Baptista LP. Metodología de la Investigación Experimental. Varela F, editor. La Habana; 2010.

[22] Virginia SL, Zayas CA. Metodología de la Investigación Científica. Centro de Estudios de la Educación Superior "Manuel F. Gran”. Universidad de Oriente; 1998.

[23] Tapia B, María A. Apuntes "Metodología de Investigación”. Ingeniería en Gestión Informática. Santiago de Cuba; 2000.

[24] García C, José E. Notas de Clase para la auto-preparación sobre conceptos básicos de los elementos componentes del diseño de la investigación y generalidades del diseño del experimento. Ciudad de La Habana: IPSJAE; 2003. 